\title{
Origin of micrometer-scale propagation lengths of heat-carrying acoustic excitations in amorphous silicon
}

\author{
Taeyong Kim $\odot,{ }^{1}$ Jaeyun Moon, ${ }^{2}$ and Austin J. Minnich ${ }^{1, *}$ \\ ${ }^{1}$ Division of Engineering and Applied Science, California Institute of Technology, Pasadena, California 91125, USA \\ ${ }^{2}$ Department of Materials Science and Engineering, University of Tennessee, Knoxville, Tennessee 37996, USA
}

(Received 22 July 2020; revised 5 March 2021; accepted 27 April 2021; published 9 June 2021)

\begin{abstract}
The heat-carrying acoustic excitations of amorphous silicon are of interest because their mean free paths may approach micron scales at room temperature. Despite extensive investigation, the origin of the weak acoustic scattering in the heat-carrying frequencies remains a topic of debate. Here, we report measurements of the thermal conductivity mean free path accumulation function in amorphous silicon thin films from 60 to $315 \mathrm{~K}$ using transient grating spectroscopy. With additional picosecond acoustics measurements and considering the power-law frequency dependence of scattering mechanisms in glasses, we reconstruct the mean free paths from $\sim 0.1-3 \mathrm{THz}$. The mean free paths are independent of temperature and exhibit a Rayleigh scattering trend over most of this frequency range. The observed trend is inconsistent with the predictions of numerical studies based on normal mode analysis but agrees with diverse measurements on other glasses. The micron-scale MFPs in amorphous $\mathrm{Si}$ arise from the absence of anharmonic or thermally activated relaxation damping in the sub- $\mathrm{THz}$ frequencies, leading to heat-carrying acoustic excitations with room-temperature MFPs comparable to those of other glasses at cryogenic temperatures.
\end{abstract}

DOI: 10.1103/PhysRevMaterials.5.065602

\section{INTRODUCTION}

The collective acoustic excitations of amorphous solids are of fundamental interest due to their anomalous properties compared to those of crystalline solids, including an excess heat capacity at cryogenic temperatures [1,2] and scattering of acoustic excitations by two-level systems [3-7]. The dispersion and scattering of acoustic excitations responsible for heat transport have been extensively explored in many glasses using experimental methods such as inelastic scattering [8-13], tunnel junction spectroscopy [14], Brillouin scattering [15-17], and picosecond acoustics [18,19], among others. These studies have generally found that excitations with well-defined frequency and wave vector are supported up to $\sim 1 \mathrm{THz}$. The mean free path versus frequency is observed to exhibit clear power laws [20,21], indicating the dominance of a single scattering mechanism within a given frequency band. Considering temperatures above $20 \mathrm{~K}$, these scattering mechanisms include Akhiezer damping, thermally activated relaxation damping, and Rayleigh scattering [3,5].

Amorphous silicon $(\mathrm{aSi})$ is an interesting glass for several reasons. First, at ultrasonic frequencies, attenuation by twolevel systems is observed in vitreous silica but not in aSi, suggesting a low density of these systems in aSi [22,23]. Second, thermal transport measurements indicate that the thermal conductivity of aSi can be higher than those of many glasses [24-27] and that heat-carrying acoustic excitations travel distances on the order of one micron at room temperature despite the atomic disorder [27-30]. For comparison, the value in-

\footnotetext{
*aminnich@caltech.edu
}

ferred for vitreous silica at room temperature is on the order of a few nanometers [21,31].

Experimentally probing the scattering of acoustic excitations in this regime with small sample volume is a long-standing experimental challenge, and as a result, studies of the acoustic excitations in aSi relied on numerical simulations based on normal mode analysis. Feldman and Allen classified the excitations in aSi as propagons, diffusons, and locons according to the qualities of the normal mode eigenvectors [32]. Fabian and Allen computed anharmonic decay rates of the normal modes of aSi, predicting that they should exhibit a clear temperature dependence [33]. Other molecular dynamics simulations based on normal mode analysis predicted that the MFPs decrease as $\omega^{-2}$ for few $\mathrm{THz}$ excitations, leading to the conclusion that they are damped by anharmonicity [34,35]. Although some of these predictions are reported to be consistent with the experiment [36], others are not. For instance, the predicted temperature dependence of $\mathrm{THz}$ excitations in Ref. [33] is not observed experimentally using inelastic x-ray scattering [9]. In the hypersonic frequency band $\sim 100 \mathrm{GHz}$, the measured values of attenuation are lower than those predicted by anharmonic damping [37].

An experimental approach to constrain the frequencydependence of sub-THz phonon scattering in aSi would help to resolve this discrepancy. For solids like aSi with MFPs in the micron range, transient grating (TG) is a tabletop experimental method that is capable of measuring the MFP accumulation function, or the cumulative thermal conductivity distribution versus MFP [38]. The technique relies on observations of nondiffusive thermal transport to constrain this function. The frequency-dependent MFPs and hence the scattering mechanisms can be reconstructed from 
(a)

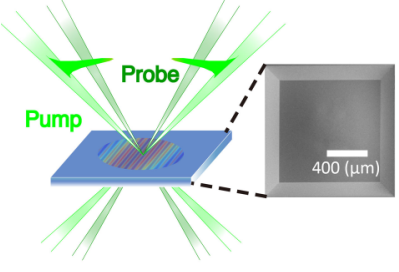

(b)

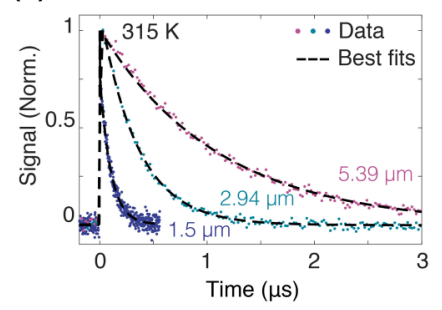

FIG. 1. (a) Schematic of the TG experiment. Two pump laser pulses are interfered on the sample, impulsively creating a spatially periodic temperature rise. The probe beams diffract from the transient grating, monitoring the thermal relaxation. A scanning electron microscope image of the free-standing amorphous silicon membrane is shown. (b) Representative measured signals versus time and single exponential fits for aSi at $315 \mathrm{~K}$ for various grating periods.

these measurements with additional information. First, the frequency-dependent MFPs in other glasses exhibit clear power laws $[8,20,39]$, and such power laws may be reasonably assumed to occur in aSi as well. Second, additional data on the acoustic dispersion and scattering are known for aSi. Specifically, the dispersion of acoustic excitations is isotropic and experimentally available [9], and the low and high frequency limits of the attenuation coefficient are known from picosecond acoustics [37] and inelastic x-ray scattering [9], respectively. Collectively, this information provides ample constraints for the reconstruction.

Here, we report the application of this approach to reconstruct the MFPs of sub- $\mathrm{THz}$ acoustic excitations in a free-standing aSi thin film. The MFPs are independent of temperature and exhibit a Rayleigh-type scattering trend over most of the accessible frequency range from $\sim 0.1-3 \mathrm{THz}$. These trends are inconsistent with predictions from normal mode analysis but agree with the trends measured in other glasses. The distinguishing feature of aSi is the weak anharmonic or thermally activated relaxation damping of sub- $\mathrm{THz}$ vibrations, leading to acoustic attenuation in aSi at room temperature comparable to that of vitreous silica at $\sim 1 \mathrm{~K}$.

\section{EXPERIMENT}

We used TG to measure the thermal diffusivity of $\sim 500$-nm-thick, free-standing aSi thin films with variable grating period $L$ from $\sim 0.75-15.7 \mu \mathrm{m}$ and at several temperatures from 60 to $315 \mathrm{~K}$. The samples were prepared using the method of Ref. [9]. Amorphous silicon was deposited on a silicon nitride handle wafer using plasma-enhanced chemical vapor deposition (PECVD) with silane gas diluted (5\%) in argon gas at a deposition table temperature of $470 \mathrm{~K}$. The expected hydrogen content is $\sim 20 \%$ [9], which was reported not to measurably affect the thermal transport [40] (see also Sec. II of Ref. [9]). The thickness of the aSi layer was $500 \mathrm{~nm}$ as measured by spectroscopic reflectometry (Filmetrics model F40). The handle wafer was subsequently etched using reactive-ion dry etching for 10 minutes, resulting in a freestanding aSi film with a rectangular aperture of area $\sim 1 \mathrm{~mm}^{2}$.

A schematic of the TG experiment and a scanning electron microscope image of the sample are provided in Fig. 1(a);

a complete description of the TG spectroscopy experiment employed in this work can be found in Refs. [41,42]. Briefly, two pump laser pulses (wavelength $515 \mathrm{~nm}$, pulse duration $\approx 1 \mathrm{~ns}$, repetition rate $200 \mathrm{~Hz}$ ) are focused on the sample $\left(1 / e^{2}\right.$ diameter $\left.520 \mu \mathrm{m}\right)$; the optical interference creates a spatially periodic heating profile with tunable grating period $(L \sim 0.75-15.7 \mu \mathrm{m})$ and wave vector $(q=2 \pi / L)$. A continuous wave laser beam (wavelength $532 \mathrm{~nm}$, chopped at $3.2 \%$ duty cycle to minimize steady heating, $470 \mu \mathrm{m} 1 / e^{2}$ diameter) diffracts from the grating, monitoring its thermal relaxation. We employ a heterodyne detection method to increase the signal-to-noise ratio [43]. The optical powers were chosen to yield adequate signal-to-noise ratio while minimizing steady heating (see Supplementary Material [44], Sec. V for additional discussion). The thermal diffusivity was obtained using $\alpha=L^{2} /\left(4 \pi^{2} \tau\right)$ where $\tau$ is the thermal decay time constant. Finally, the thermal conductivity was computed using the literature heat capacity [45].

TG provides information on the heat carrier MFPs if they are comparable to $\sim L / 2 \pi[38,46]$. In the present case, extrinsic boundary scattering may decrease the MFP from its intrinsic value, but for specularity parameters on the order of those measured at surfaces terminating crystalline silicon $(\sim 0.95$ in Ref. [47]) the maximum MFP is expected to be $\sim 0.5(1+p) /(1-p) \sim 20 \mu \mathrm{m}$. Excitations with MFPs on this length scale should be readily detectable if present despite the boundary scattering.

\section{RESULTS}

Figure 1(b) shows representative signals at $\sim 315 \mathrm{~K}$ (additional data and fits are provided in Supplementary Material, Sec. I). The decay exhibits a single exponential profile with a time constant in the range of tens of nanoseconds to microseconds due to thermal transport. The thermal diffusivity is obtained by extracting the time constant of the single exponential decay; the thermal conductivity is then computed using the measured heat capacity from Ref. [45].

The measured TG signal for a grating period of $L=754$ $\mathrm{nm}$ is shown in Fig. 2(a). The actual thermal decay is clearly slower than that predicted based on the thermal diffusivity measured at $L \gtrsim 10 \mu \mathrm{m}$, indicating the presence of acoustic excitations with MFPs comparable to the grating period. Measurements of the decay rate versus $q^{2}$ for all the grating periods at $60 \mathrm{~K}$ are given in Fig. 2(b). The measured decay rate follows the linear trend expected from diffusion theory up to around $q^{2} \sim 4.6 \mu \mathrm{m}^{-2}$ ( $\left.L \sim 3 \mu \mathrm{m}\right)$, above which the decay rate becomes smaller.

Figure 2(c) shows the measured thermal conductivity versus grating period obtained from these time constants at $60 \mathrm{~K}$ considered in this study. As the grating period becomes comparable to some MFPs, the effective thermal conductivity varies with grating period due to the ballistic transport of the acoustic excitations over the scale of the grating period. For $L=754 \mathrm{~nm}$, the reduction of the thermal conductivity from the value at $L=10.7 \mu \mathrm{m}$ is $\sim 30 \%$.

We calculated the MFP accumulation function that is consistent with these measurements using the method of Ref. [42]. More precisely, the posterior probability distribution of the thermal conductivity accumulation function 
(a)

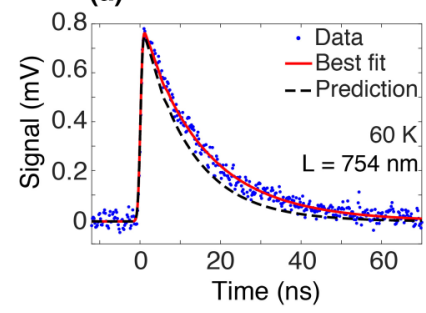

(b)

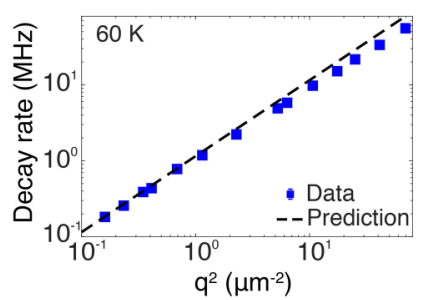

(c)

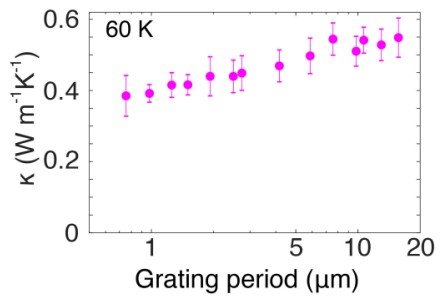

FIG. 2. (a) Measured TG signal versus time (symbols) for grating period $L=754 \mathrm{~nm}\left(q^{2} \sim 70 \mu \mathrm{m}^{-2}\right)$ at $T=60 \mathrm{~K}$, along with the best fit (solid red line) and the predicted decay using the thermal conductivity measured at $L=10.7 \mu \mathrm{m}$ (dashed black line). The actual signal decays slower than predicted, indicating a departure from diffusive thermal transport. (b) Inverse time constant versus $q^{2}$ at $T=60 \mathrm{~K}$. A deviation from the linear trend is observed for $L \lesssim 3 \mu \mathrm{m}\left(q^{2} \gtrsim 4.6 \mu \mathrm{m}\right)$. (C) Measured thermal conductivity versus grating period at $T=60 \mathrm{~K}$. A decrease in thermal conductivity of $\sim 30 \%$ is observed.

along with credible intervals were obtained for each temperature using Bayesian inference with a Metropolis-Hastings Markov chain Monte Carlo algorithm. The posterior distribution bounded by the $99 \%$ credible interval at $60 \mathrm{~K}$ is shown as the shaded region in Fig. 3(a). The figure shows that the

(a)

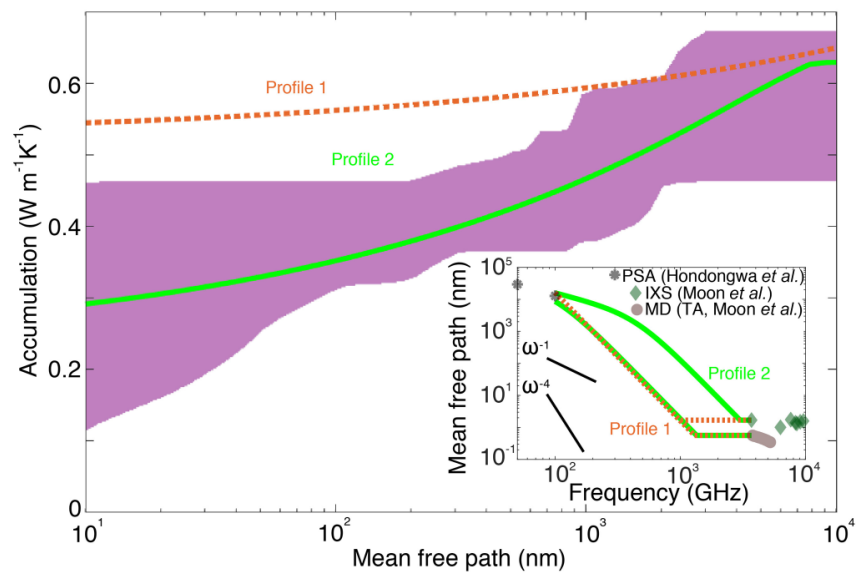

(b)

(c)
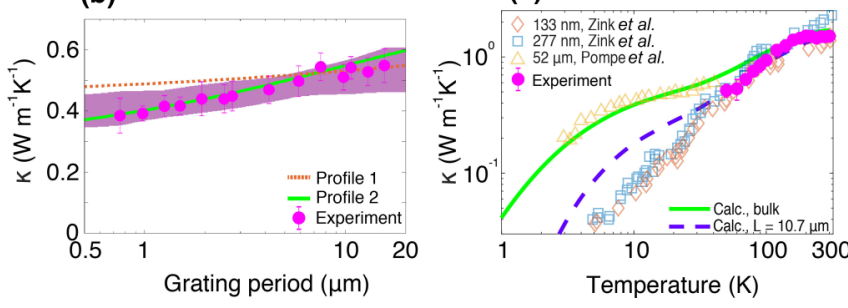

FIG. 3. (a) Posterior probability distribution and $99 \%$ credible interval of the thermal conductivity accumulation versus the mean free path at $60 \mathrm{~K}$ (shaded region), along with the predicted thermal conductivity accumulation at $L=10.7 \mu \mathrm{m}$ from each candidate profile (lines). Inset: Candidate MFP profiles versus frequency: $\omega^{-4}$, constant (profile 1: dotted lines); $\omega^{-1}, \omega^{-4}$, constant (profile 2: solid lines). Upper and lower curves indicate the LA and TA MFPs, respectively. (b) Measured thermal conductivity versus the grating period (symbols) along with that predicted from posterior distribution (shaded region) and the two profiles (lines) at $60 \mathrm{~K}$. (c) Measured thermal conductivity versus temperature for $L=10.7 \mu \mathrm{m}$ (filled circles) along with the literature data (open symbols). The calculated bulk $(L=10.7 \mu \mathrm{m})$ thermal conductivities using profile 2 are shown as the solid (dashed) line. percentage of the heat carried by excitations with MFPs larger than $1 \mu \mathrm{m}$ is $\sim 31 \pm 18 \%$, qualitatively agreeing with the recent observation that more than $50 \%$ of the heat is carried by MFPs exceeding $100 \mathrm{~nm}$ [30].

We now examine the constraints that the thermal conductivity accumulation function places on the allowed frequency dependence of the MFPs. The strategy is to construct a lowenergy Debye model for the thermal conductivity and identify the frequency-dependent MFPs that can simultaneously explain the accumulation function and independent data from inelastic x-ray scattering (IXS), picosecond acoustics, and other sources. Following Ref. [38], the measured thermal conductivity $\kappa_{i}$ can be expressed as

$$
\kappa_{i}=\sum_{s} \int_{0}^{\omega_{m, s}} S\left(x_{i, s}\right)\left[\frac{1}{3} C_{s}(\omega) v_{s} \Lambda_{s}(\omega)\right] d \omega+\kappa_{I R}(T),
$$

where $s$ indexes the polarization, $q_{i}=2 \pi L_{i}^{-1}, x_{i, s}=q_{i} \Lambda_{s}(\omega)$, $S\left(x_{i, s}\right)$ is the isotropic suppression function in Refs. [38,48], $\omega_{m, s}$ is the cutoff frequency for collective acoustic excitations, and $\kappa_{I R}(T)$ is the contribution from excitations above the Ioffe-Regel (IR) cutoff frequency. The Debye heat capacity $C_{s}$ of acoustic excitations is calculated from the group velocities $v_{s}$ which are known, isotropic, and independent of temperature [9,49]. The first term of Eq. (1) is a Debye model for the thermal conductivity of an isotropic solid that includes the effect of nondiffusive thermal transport over a grating period.

The desired quantity is $\Lambda_{s}(\omega)$, or the MFP versus frequency for the LA and TA polarizations. The grating period dependence of the thermal conductivity provides constraints on $\Lambda_{s}(\omega)$, and independent data provide further constraints. First, the linewidths of LA excitations at frequencies above $3.7 \mathrm{THz}$ are known from IXS measurements and are independent of temperature [9]. As the TA linewidths are not accessible with IXS, we use the values from MD simulations as these values for the LA branch quantitatively agreed with IXS measurements. These values allow the thermal conductivity of collective excitations above $\sim 3.7 \mathrm{THz}$ to be obtained from Eq. (1); at room temperature this contribution is $\sim 0.5 \mathrm{Wm}^{-1} \mathrm{~K}^{-1}$.

Second, the attenuation lengths at hypersonic frequencies $\sim 100 \mathrm{GHz}$ can be obtained from picosecond acoustics from 30 to $300 \mathrm{~K}$. The room temperature value is available in Ref. [37]. To obtain the value at other temperatures, we deposited aSi films using the same method as for the TG samples 
but on a sapphire wafer and with variable thickness between 2 to $6 \mu \mathrm{m}$. Measurements of the amplitude of a thermoelastic strain pulse as it makes multiple round trips through the film allow the intrinsic attenuation lengths to be extracted (see Supplementary Material, Sec. III for further details). The measured values are on the order of 10 to $20 \mu \mathrm{m}$ and depend weakly on temperature. Although the acoustic properties of aSi thin films may differ depending on fabrication recipe, the good agreement between our room temperature value and that of Ref. [37] suggest that our films are representative of those studied in the field.

The lack of temperature dependence of the MFPs at both $100 \mathrm{GHz}$ and $\sim 3 \mathrm{THz}$ suggests that the MFPs at intermediate frequencies should also be independent of temperature. Qualitatively, these observations indicate that anharmonic or thermally activated relaxation damping are weak in aSi as they both exhibit a temperature dependence. Quantitatively, the weak temperature dependence, the PSA and IXS measurements, the measured thermal conductivity versus grating period at the five temperatures in this study, and the clear power-law frequency dependence of MFPs observed in other glasses [20] impose tight constraints on the frequencydependence of the MFPs and hence the scattering mechanisms in the sub-THz frequencies. The inset of Fig. 3(a) shows two candidate MFP profiles that satisfy these constraints for the LA and TA branches. Power-law dependencies $\Lambda \sim \omega^{-n}$ are assumed and combined using Matthiessen's rule. Profile 1 transitions from a constant value to $n=4$ corresponding to Rayleigh scattering, while profile 2 transitions from constant to $n=4$ at $\sim 2-3 \mathrm{THz}$ and then to $n=1$ at $\sim 200-300 \mathrm{GHz}$.

The thermal conductivity accumulation versus MFP at $60 \mathrm{~K}$ computed from these profiles is given in Fig. 3(a). In this figure, $\kappa_{I R}$ was chosen to match the measured thermal conductivity for $L \gtrsim 10 \mu \mathrm{m}$ to facilitate comparison. Profile 2 exhibits better agreement with the posterior distribution, with profile 1 exhibiting a weaker trend with MFP compared to the posterior distribution.

The thermal conductivity versus grating period for the posterior distribution and both profiles is shown in Fig. 3(b). Profile 2 again yields better agreement with the trend of thermal conductivity versus grating period compared to profile 1. An alternate profile that increases as $n=4$ immediately at $3.7 \mathrm{THz}$ yields a thermal conductivity that exceeds the experimental values at all temperatures (not shown). We find that profiles consistent with profile 2 are best able to explain the magnitude and grating period dependence of the thermal conductivity. Specifically, the MFPs must remain constant as frequency decreases and then increase rapidly as $n=4$. To agree with the PSA data, the trend must then switch to $n=1$ or $n=2$. The $n=1$ trend is favored by independent picosecond acoustic measurements at $50 \mathrm{GHz}$ and $100 \mathrm{GHz}$ [37]. Considering the weak dependence of the MFPs on temperature, this discussion suggests a structural origin of scattering at $\sim 100 \mathrm{GHz}$ such as density fluctuations, which is predicted to exhibit an $n=1$ trend [50].

We provide further evidence in support of profile 2 by calculating the bulk thermal conductivity versus temperature with these MFPs and comparing it to the measured values in the present work and Refs. [36,51]. Figure 3(c) shows that the computed bulk thermal conductivity agrees with the mea- surements of Ref. [51]. However, discrepancies are observed between the computed bulk thermal conductivity and the data of this work and Ref. [36]. Accounting for the suppression of the contribution of phonons of MFP exceeding the maximum grating period used in the present experiments $(L=$ $10.7 \mu \mathrm{m}$ ) yields good agreement with our data and qualitative agreement with Ref. [36]. Therefore, the trends of their measurements could be attributed to extrinsic boundary scattering limiting the maximum MFP rather than intrinsic scattering mechanisms. The value of specularity parameter required to produce micron-scale MFPs $\Lambda$ for the $d \sim 100$-nm-thick membrane used in their work is $\sim 0.95$; this specularity agrees well with that of Ref. [47].

Evidence in support of the $n=1$ trend at $\sim 100 \mathrm{GHz}$ can be obtained by extrapolating the MFP inferred from the dominant phonon approximation with the data of Ref. [51], assuming that the physical origin of the scattering remains the same at temperatures below $10 \mathrm{~K}$. The dominant heat-carrying phonon frequency at $\sim 3 \mathrm{~K}$ is $\sim 260 \mathrm{GHz}$ [31]; taking their measured thermal conductivity and the computed heat capacity of acoustic excitations at $3 \mathrm{~K} \quad\left(\kappa \sim 0.2 \mathrm{~W} \mathrm{~m}^{-1} \mathrm{~K}^{-1}\right.$, and $C \sim 48 \mathrm{Jm}^{-3} \mathrm{~K}^{-1}$, respectively) along with the average sound velocity $\left(v \sim 4400 \mathrm{~ms}^{-1}\right)$, the average MFP of the dominant phonon can be obtained using $\Lambda_{\text {dom }} \sim 3 \kappa / C v \approx 3 \mu \mathrm{m}$. If the MFP trend follows $n=1$ at these frequencies, the MFP from Ref. [51] at $3 \mathrm{~K}$ implies a MFP of $\sim 10-20 \mu \mathrm{m}$ at 50 to $100 \mathrm{GHz}$, close to the PSA measurements of the present work. This analysis thus supports the magnitude, frequency dependence, and weak temperature dependence of the reconstructed MFPs.

We now reconstruct the MFP profile that best explains the data and is consistent with profile 2. Given the above constraints, the MFPs are characterized by only two parameters: the transition frequencies from $n=4$ to $n=1$ for both acoustic polarizations, $\omega_{m, L}$ and $\omega_{m, T}$. The remaining unknown parameter is $\kappa_{I R}(\mathrm{~T})$, which may depend on temperature. We let this function follow the temperature dependence of the heat capacity, $\kappa_{I R}(T) \propto C(T)$ [52]. Then, we obtain the MFPs that best explain the TG data by numerically optimizing these parameters to fit the TG data at all temperatures. The comparison between the computed and measured thermal conductivity is provided in Supplementary Material, Sec. II, and good agreement is observed at all temperatures. Further discussion of the choices of $\omega_{m, L}$, $\omega_{m, T}$, and $\kappa_{I R}$ that are compatible with the data is given in Supplementary Material, Sec. IV; the trend of MFP with frequency remains the same for all of these parameter sets.

The reconstructed MFPs for the LA and TA polarizations are shown in Fig. 4. To gain physical insight into the scattering mechanisms in aSi, we compare the characteristics of the reconstructed MFPs with those reported for vitreous silica, an extensively studied glass with representative measurements provided in Fig. 4. The frequency dependence of the MFPs in aSi agrees well with these and other measurements [8,14,16-19,53,54]. The $n=4$ Rayleigh scattering trend for both glasses occurs in the 1-3 THz range with a transition to a weaker power law in the sub- $\mathrm{THz}$ frequencies. Thus, the frequency dependence of acoustic scattering in aSi shares several features in common with other glasses. 


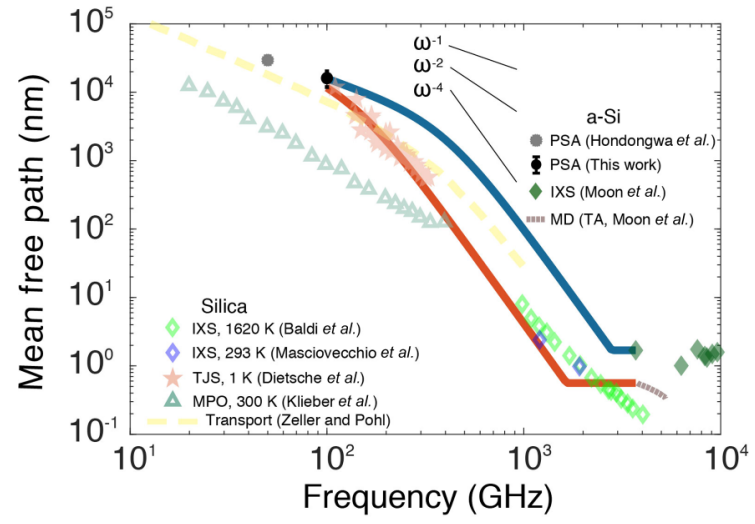

FIG. 4. Reconstructed MFPs versus frequency for thermal acoustic excitations in aSi at $300 \mathrm{~K}$. Also shown are PSA data for aSi (Ref. [37]), literature data for vitreous silica from inelastic x-ray scattering (diamonds, Refs. [8,55]), tunnel junction spectroscopy (5-pointed stars, Ref. [14]), a multipulse optical technique (upward pointing triangles, Ref. [39]), and from transport measurements (dashed line, Ref. [31]).

However, comparing the attenuation between vitreous silica and aSi, differences emerge. First, acoustic scattering in aSi exhibits little temperature dependence. Although the authors of Ref. [36] reported a $T^{-2}$ dependence of the MFP, their measurements may have been affected by extrinsic boundary scattering as discussed above. In contrast, the scattering in vitreous silica exhibits a strong temperature dependence as observed in Fig. 4, highlighting the lack of anharmonic or thermally-activated relaxation in aSi.

Other differences become clear on closer examination. Comparing the LA MFPs at $\sim 1-2 \mathrm{THz}$, the attenuation due to Rayleigh scattering is weaker in aSi by around a factor of 5 , expected as aSi is a monatomic glass with less atomic disorder. Further, at room temperature the $n=4$ trend yields to a $n=1$ trend at $\sim 700 \mathrm{GHz}$ in vitreous silica while the same transition occurs at $\sim 300-400 \mathrm{GHz}$ in aSi. At cryogenic temperatures $\sim 1 \mathrm{~K}$ for vitreous silica, the transition frequencies in both materials are comparable. This difference again indicates weaker damping by anharmonic or thermally activated relaxation in aSi and has an important consequence: excitations with MFPs in the micron range occur at frequencies of $\sim 200-1000 \mathrm{GHz}$ in aSi versus $\lesssim 100 \mathrm{GHz}$ in vitreous silica at room temperature owing to the steep $n=4$ slope of Rayleigh scattering. The spectral heat capacity $C_{\omega}=$ $\hbar \omega D(\omega)\left(\partial f_{B E} / \partial T\right) \sim \omega^{2}$ for $\hbar \omega / k_{B} T \ll 1$, which is satisfied in the sub- $\mathrm{THz}$ frequencies at room temperature. Therefore, considering the different frequencies of micron-scale MFP excitations in the two materials $\left(\omega_{\mathrm{SiO}_{2}} \sim 100 \mathrm{GHz}\right.$ and $\omega_{a S i} \sim 1$ $\mathrm{THz}$ ), the heat carried by those in aSi is larger by a factor of $\left(\omega_{a S i} / \omega_{\mathrm{SiO}_{2}}\right)^{2} \sim 100$. The result is that in aSi, heat-carrying excitations at room temperature have micron-scale MFPs, while the MFPs of excitations in the same frequency band for vitreous silica are smaller by an order of magnitude. Excitations in vitreous silica with micron-scale MFPs have too low frequency to transport substantial heat at room temperature. Remarkably, the scattering rates observed in aSi are of the same order as those measured in vitreous silica at $1 \mathrm{~K}$ [14], highlighting the unusually weak acoustic scattering in aSi.

\section{DISCUSSION}

Our conclusions on the origin of weak scattering of sub$\mathrm{THz}$ excitations in aSi are consistent with these prior studies of other glasses but not with the conclusions of numerical studies of excitations below the IR frequency [33-35,56,57]. In these studies, the Hamiltonian for atoms in a supercell is diagonalized in the harmonic approximation to yield normal modes. The original studies of Allen and Feldman used these normal modes to classify excitations in glasses as propagons, diffusons, and locons depending on the properties of the eigenvectors [52]. The lifetimes of these modes are obtained using normal mode decomposition and molecular dynamics [58,59]. With these approaches, these studies have generally concluded that scattering in aSi varies as $\omega^{-2}$ for frequencies around $\sim 2 \mathrm{THz}$ and below. From this trend, the scattering mechanism has been postulated to involve anharmonicity [34,35] and to exhibit a temperature dependence [33].

We first address the classification of acoustic excitations. Various numerical [32-34] and experimental works [29] noted a transition in the character of vibrations in $\mathrm{aSi}$ around $\sim 1-2 \mathrm{THz}$, leading to the introduction of "diffusons" as nonpropagating yet delocalized vibrations in Refs. [32,33]. In contrast, our work attributes this transition to a change in frequency-dependent MFPs of collective acoustic excitations. The crossover from propagons and diffusons at $\sim 1-2 \mathrm{THz}$ coincides with the transition from Rayleigh scattering to the Kittel regime in the present work and thus can be explained without the definition of a new type of vibration. The IR crossover for the transition from collective excitations to incoherent excitations, which occurs well above 1 to $2 \mathrm{THz}$ in amorphous $\mathrm{Si}$, is sufficient to describe the different characters of excitations in glasses.

The second discrepancy is the prediction by normal-mode analysis of the frequency-dependence $(n=2)$ and anharmonic origin of scattering in the few $\mathrm{THz}$ frequency range. Specifically, the MFPs predicted from normal mode analysis are on the order of 10 to $20 \mathrm{~nm}$ at $\sim 1 \mathrm{THz}$ and vary as $\omega^{-2}$ (see Fig. 4(b) of Ref. [34]), which cannot explain the measurements of the present work. In particular, extrapolating the $20 \mathrm{~nm}$ value at $1 \mathrm{THz}$ to $100 \mathrm{GHz}$ as $\omega^{2}$ yields $\sim 2 \mu \mathrm{m}$, which is smaller by a factor of 10 compared to the PSA measurements at the same frequency (10 to $20 \mu \mathrm{m}$, Ref. [37] and this work). Here, the discrepancy appears to arise from the implicit assumption of the normal mode decomposition that the heat-carrying excitations of glasses are the normal modes of the supercell. This assumption is not compatible with basic many-body physics and scattering theory, which instead gives the proper definition and lifetime of a collective excitation of a many-body system using the self-energy and the single-particle Green's function [60]. Rather than normal modes, a physical picture of acoustic excitations of a glass that is compatible with this framework is that originally postulated by Kittel [61] in the continuum limit and later by Zeller and Pohl [31], in which a glass is imagined to consist of a fictitious atomic lattice along with perturbations representing the mass and force constant disorder in the actual glass. The undamped excitations of the fictitious atomic lattice acquire a lifetime owing to the disorder of the actual glass. The dispersion and lifetimes of these excitations can be measured experimentally 
using inelastic scattering, as has been performed for many glasses in the past decades [8-13]. In contrast, the lifetimes of normal modes do not appear to be experimentally accessible or physically meaningful as they are unable to explain the thermal conductivity measurements presented here.

\section{CONCLUSION}

In summary, we report measurements of the thermal conductivity accumulation function of aSi and reconstruction of the MFPs versus frequency using picosecond acoustics and transient grating spectroscopy. The reconstructed MFPs lack a temperature dependence and exhibit a trend characteristic of structural scattering by point defects and density fluctuations.
This result is at variance with numerical studies based on normal mode analysis but is broadly consistent with prior studies of vitreous silica and other glasses. The micron-scale MFPs of heat-carrying excitations at room temperature are found to arise from the weak anharmonic and thermally activated relaxation damping of sub- $\mathrm{THz}$ excitations, leading to room temperature scattering comparable to that of other glasses at cryogenic temperatures.

\section{ACKNOWLEDGMENTS}

The authors acknowledge discussions with A. B. Robbins and B. C. Daly. This work was supported by the 2018 GISTCaltech Research Collaboration.
[1] V. K. Malinovsky, V. N. Novikov, P. P. Parshin, A. P. Sokolov, and M. G. Zemlyanov, Europhys. Lett. 11, 43 (1990).

[2] X. Liu and H. v Löhneysen, Europhys. Lett. 33, 617 (1996).

[3] J. Jäckle, L. Piché, W. Arnold, and S. Hunklinger, J. Non-Cryst. Solids 20, 365 (1976).

[4] W. A. Phillips, J. Low Temp. Phys. 7, 351 (1972).

[5] S. Hunklinger and W. Arnold, Physical Acoustics, Vol. 12 (Academic, New York, 1976), pp. 155-215.

[6] P. W. Anderson, B. I. Halperin, and C. M. Varma, Philos. Mag.: A J. Theor. Exp. Appl. Phys. 25, 1 (1972).

[7] M. P. Zaitlin and A. C. Anderson, Phys. Status Solidi (b) 71, 323 (1975).

[8] G. Baldi, V. M. Giordano, G. Monaco, and B. Ruta, Phys. Rev. Lett. 104, 195501 (2010).

[9] J. Moon, R. P. Hermann, M. E. Manley, A. Alatas, A. H. Said, and A. J. Minnich, Phys. Rev. Mater. 3, 065601 (2019).

[10] G. Monaco and V. M. Giordano, Proc. Natl. Acad. Sci. USA 106, 3659 (2009).

[11] B. Rufflé, G. Guimbretière, E. Courtens, R. Vacher, and G. Monaco, Phys. Rev. Lett. 96, 045502 (2006).

[12] C. Masciovecchio, G. Ruocco, F. Sette, M. Krisch, R. Verbeni, U. Bergmann, and M. Soltwisch, Phys. Rev. Lett. 76, 3356 (1996).

[13] F. Sette, M. H. Krisch, C. Masciovecchio, G. Ruocco, and G. Monaco, Science 280, 1550 (1998).

[14] W. Dietsche and H. Kinder, Phys. Rev. Lett. 43, 1413 (1979).

[15] R. Vacher, H. Sussner, and S. Hunklinger, Phys. Rev. B 21, 5850 (1980).

[16] R. Vacher and J. Pelous, Phys. Rev. B 14, 823 (1976).

[17] P. Benassi, S. Caponi, R. Eramo, A. Fontana, A. Giugni, M. Nardone, M. Sampoli, and G. Viliani, Phys. Rev. B 71, 172201 (2005).

[18] T. C. Zhu, H. J. Maris, and J. Tauc, Phys. Rev. B 44, 4281 (1991).

[19] A. Devos, M. Foret, S. Ayrinhac, P. Emery, and B. Rufflé, Phys. Rev. B 77, 100201(R) (2008).

[20] J. J. Freeman and A. C. Anderson, Phys. Rev. B 34, 5684 (1986).

[21] K. E. Goodson, M. I. Flik, L. T. Su, and D. A. Antoniadis, J. Heat Transfer 116, 317 (1994).

[22] M. Von Haumeder, U. Strom, and S. Hunklinger, Phys. Rev. Lett. 44, 84 (1980).
[23] X. Liu, B. E. White, Jr., R. O. Pohl, E. Iwanizcko, K. M. Jones, A. H. Mahan, B. N. Nelson, R. S. Crandall, and S. Veprek, Phys. Rev. Lett. 78, 4418 (1997).

[24] L. Wieczorek, H. J. Goldsmid, and G. L. Paul, Thermal conductivity of amorphous films, in Thermal Conductivity 20, edited by D. P. H. Hasselman and J. R. Thomas (Springer, Boston, 1989), pp. 235-241.

[25] B. S. W. Kuo, J. C. M. Li, and A. W. Schmid, Appl. Phys. A 55, 289 (1992).

[26] S. Moon, M. Hatano, M. Lee, and C. P. Grigoropoulos, Int. J. Heat Mass Transfer 45, 2439 (2002).

[27] X. Liu, J. L. Feldman, D. G. Cahill, R. S. Crandall, N. Bernstein, D. M. Photiadis, M. J. Mehl, and D. A. Papaconstantopoulos, Phys. Rev. Lett. 102, 035901 (2009).

[28] S. Kwon, J. Zheng, M. C. Wingert, S. Cui, and R. Chen, ACS Nano 11, 2470 (2017).

[29] J. L. Braun, C. H. Baker, A. Giri, M. Elahi, K. Artyushkova, T. E. Beechem, P. M. Norris, Z. C. Leseman, J. T. Gaskins, and P. E. Hopkins, Phys. Rev. B 93, 140201(R) (2016).

[30] Y. Pan, J. Zhou, and G. Chen, Phys. Rev. B 101, 144203 (2020).

[31] R. C. Zeller and R. O. Pohl, Phys. Rev. B 4, 2029 (1971).

[32] P. B. Allen, J. L. Feldman, J. Fabian, and F. Wooten, Philos. Mag. B 79, 1715 (1999).

[33] J. Fabian and P. B. Allen, Phys. Rev. Lett. 77, 3839 (1996).

[34] J. M. Larkin and A. J. H. McGaughey, Phys. Rev. B 89, 144303 (2014).

[35] K. Sääskilahti, J. Oksanen, J. Tulkki, A. J. H. McGaughey, and S. Volz, AIP Adv. 6, 121904 (2016).

[36] B. L. Zink, R. Pietri, and F. Hellman, Phys. Rev. Lett. 96, 055902 (2006).

[37] D. B. Hondongwa, B. C. Daly, T. B. Norris, B. Yan, J. Yang, and S. Guha, Phys. Rev. B 83, 121303(R) (2011).

[38] A. J. Minnich, Phys. Rev. Lett. 109, 205901 (2012).

[39] C. Klieber, E. Peronne, K. Katayama, J. Choi, M. Yamaguchi, T. Pezeril, and K. A. Nelson, Appl. Phys. Lett. 98, 211908 (2011).

[40] D. G. Cahill, M. Katiyar, and J. R. Abelson, Phys. Rev. B 50, 6077 (1994).

[41] N. K. Ravichandran, H. Zhang, and A. J. Minnich, Phys. Rev. X 8, 041004 (2018).

[42] A. B. Robbins, S. X. Drakopoulos, I. Martin-Fabiani, S. Ronca, and A. J. Minnich, Proc. Natl. Acad. Sci. USA 116, 17163 (2019). 
[43] A. A. Maznev, K. A. Nelson, and J. A. Rogers, Opt. Lett. 23, 1319 (1998).

[44] See Supplemental Material at http://link.aps.org/supplemental/ 10.1103/PhysRevMaterials.5.065602 for more information (i) additional representative raw transient grating signals, (ii) thermal conductivity versus grating period at $315 \mathrm{~K}-80 \mathrm{~K}$ along with calculation, (iii) additional details on picosecond acoustics (PSA), (iv) additional information regarding the crossover frequencies and thermal conductivity above Ioffe-Regel crossover that are compatible with the data, (v) Details concerning selfheating and the optical power..

[45] D. R. Queen, X. Liu, J. Karel, T. H. Metcalf, and F. Hellman, Phys. Rev. Lett. 110, 135901 (2013).

[46] J. A. Johnson, A. A. Maznev, J. Cuffe, J. K. Eliason, A. J. Minnich, T. Kehoe, C. M. S. Torres, G. Chen, and K. A. Nelson, Phys. Rev. Lett. 110, 025901 (2013).

[47] T. Klitsner and R. O. Pohl, Phys. Rev. B 36, 6551 (1987).

[48] A. A. Maznev, J. A. Johnson, and K. A. Nelson, Phys. Rev. B 84, 195206 (2011).

[49] J. Moon, B. Latour, and A. J. Minnich, Phys. Rev. B 97, 024201 (2018).

[50] D. Walton, Solid State Commun. 14, 335 (1974).
[51] G. Pompe and E. Hegenbarth, Phys. Status Solidi (b) 147, 103 (1988).

[52] J. L. Feldman, M. D. Kluge, P. B. Allen, and F. Wooten, Phys. Rev. B 48, 12589 (1993).

[53] M. P. Zaitlin and A. C. Anderson, Phys. Rev. B 12, 4475 (1975).

[54] J. E. Graebner, B. Golding, and L. C. Allen, Phys. Rev. B 34, 5696 (1986).

[55] C. Masciovecchio, G. Ruocco, F. Sette, P. Benassi, A. Cunsolo, M. Krisch, V. Mazzacurati, A. Mermet, G. Monaco, and R. Verbeni, Phys. Rev. B 55, 8049 (1997).

[56] Y. He, D. Donadio, and G. Galli, Appl. Phys. Lett. 98, 144101 (2011).

[57] J. Fabian and P. B. Allen, Phys. Rev. Lett. 82, 1478 (1999).

[58] A. J. C. Ladd, B. Moran, and W. G. Hoover, Phys. Rev. B 34, 5058 (1986).

[59] A. J. H. McGaughey and M. Kaviany, Phys. Rev. B 69, 094303 (2004).

[60] P. Coleman, Introduction to Many-Body Physics (Cambridge University Press, Cambridge, England, 2015).

[61] C. Kittel, Phys. Rev. 75, 972 (1949). 